\title{
Magnetization of a nonferromagnetic metal spacer sandwiched between two magnetically ordered layers
}

\author{
V.Yu. Gorobets \\ Institute of Magnetism of the National Academy of Sciences of Ukraine \\ 36-b Vernadsky Ave. Kiev 03142, Ukraine \\ E-mail: gorob@mail.kar.net
}

Received January 6, 2004, revised April 1, 2004

\begin{abstract}
The exchange coupling of magnetically ordered layers (MOLs) through a nonmagnetic metallic spacer was calculated. The induced magnetization in the spacer, taking into account the influence of an external magnetic field, was calculated, too. This calculation shows that the energy of coupling of the MOLs through the nonmagnetic metallic spacer is a long-periodic function of the spacer's thickness and magnetic field, i.e., the exchange coupling between the layers varies from ferromagnetic to antiferromagnetic and vice versa depending on the spacer's thickness and magnetic field. Also this calculation shows that in nonferromagnetic spacer the induced magnetization can undergo many complete rotations depending on distance to the boundaries with the MOLs. Moreover, absolute value of induced magnetization nonmonotonously decays with distance from the interfaces inside the spacer. It is shown that the character of the decay of absolute value magnetization from the interfaces into the interior of the spacer is influenced by magnetic field.
\end{abstract}

PACS: 75.70.-i

In the last decade much effort has been dedicated to the study of the magnetic coupling in nanoscale multilayer systems, because ultrathin magnetic films exhibit unusual magnetic configurations and coupling not found in bulk systems $[1,2]$. In particular, in trilayer systems like ferromagnet-nonmagnetic spacer-ferromagnet (e.g., $\mathrm{Fe} / \mathrm{Cr} / \mathrm{Fe}, \mathrm{Fe} / \mathrm{Cu} / \mathrm{Fe}$, etc.) long-periodic oscillating exchange coupling as a function of spacer thickness has been found [3,4-7]. This means that the coupling changes from antiferromagnetic to ferromagnetic with spacer thickness. For deeper understanding of indirect exchange coupling through a nonmagnetic spacer one needs to investigate the magnetic properties of a nonmagnetic spacer in a multilayer system. Moreover, investigation of the magnetic properties of a nonmagnetic spacer sandwiched between ferromagnetic layers is very important not only because of oscillating exchange coupling, but also because the contact of a ferromagnetic layer with a nonmagnetic spacer must change the electronic state of the nonmagnetic spacer. Also, many experimental and theoretical works are dedicated to the study of such trilayer systems as antiferromagnet-nonmagnetic spacer-antiferromagnet. Such sys- tems attract much interest of researchers because they can be used in magneto-resistive devices, for example, in magnetic field sensors, magnetic heads in memory devices, etc. Taking into account the aforementioned problems some works are dedicated to investigation of the distribution of magnetization induced in a nonmagnetic metal spacer sandwiched between two ferromagnetic layers. Of great interest is the question of the possibility of inducing magnetization in a material which in the normal state is nonmagnetic but which can be polarized if it boundaries with a magnetically ordered material [8]. Magnetic polarization of a nonmagnetic material by a magnetically ordered material is usually called the proximity effect. The experimental study of the proximity effect is described in some works [9-11]. For example, magnetic polarization of a nonmagnetic Au spacer in multilayer systems like ferromagnet-nonmagnetic spacer-ferromagnet has been measured with the help of Mössbauer spectroscopy using probe atoms in an Au spacer [12]. A small induced magnetic moment in $\mathrm{Cu}$ at the $\mathrm{Co} / \mathrm{Cu}$ interface was detected Refs. 13,14 using circular dichroism and in [15] using NMR. The oscillating exchange coupling between two ferromagnetic layers separated by a non- 
magnetic metal spacer is explained in some theoretical works, usually using RKKY coupling [16]. Moreover, oscillatory exchange coupling between two ferromagnetic layers separated by a nonmagnetic metal spacer layer is associated with oscillation of the magnetic moment in the nonmagnetic spacer layer [3,17-19]. The magnetic moment induced in a nonmagnetic metallic spacer between two ferromagnets with magnetizations turned at an arbitrary angle is calculated theoretically in Ref. 17. As is shown in Ref. 17, the induced magnetization rotates along a complex three-dimensional spiral and can undergo many complete rotations.

In this work we propose a phenomenological method of calculating both the oscillating exchange coupling of magnetically ordered layers through a nonmagnetic metallic spacer and the induced magnetization in a the nonmagnetic spacer using a spin-density model [20] similar to the Ginzburg-Landau model [21]. In comparison with Ref.17 the approach proposed in our work allows to calculate the magnetization induced in the spacer and the oscillating exchange coupling taking into account the influence of external magnetic field. The approach can be used whether the magnetically ordered layers are ferromagnetic or antiferromagnetic. To consider the system magnetically ordered layer-nonmagnetic metallic spacer-magnetically ordered layer (MOL-NMMSMOL) in magnetic field, let us consider the case when external magnetic field $H$ is parallel to the $z$ axis and directed perpendicularly to the plane of the spacer. The interface of the spacer with the first MOL is situated in the $x y$ plane, the interface of the spacer with the second MOL is situated in the $z=L$ plane parallel to $x y$. Following Ref. 20 let us define the order parameter for the spacer as the two-component function

$$
\psi=\left(\begin{array}{c}
\Phi_{1} \\
\Phi_{2}
\end{array}\right)
$$

in terms of which the magnetic moment density of the spacer is written as

$$
\mathbf{M}=\mu_{0} \psi^{+} \hat{\boldsymbol{\sigma}} \psi,
$$

where $\mu_{0}$ is a phenomenological parameter and $\hat{\boldsymbol{\sigma}}$ are the Pauli matrices. We suppose in our approach that the state of the MOLs does not depend on distribution of the order parameter inside the spacer and is characterized only by the directions of homogenous magnetization. Using the approach of Ref. 20 and the functional method of the Ginzburg-Landau type for the order parameter $\psi$, we write the following Lagrangian function:

$$
L=\int\left[\frac{1}{2} i \hbar\left(\dot{\psi}^{+} \psi-\psi^{+} \dot{\psi}\right)-w(\psi)\right] d \mathbf{r} .
$$

Here $w$ is the energy density written to forth-order accuracy in powers of the function $\psi$ :

$\omega(\psi)=A \nabla \psi^{+} \nabla \psi+\beta \psi^{+} \psi+\frac{s}{2}\left(\psi^{+} \psi\right)^{2}-\mu_{0} H\left(\psi^{+} \hat{\sigma}_{z} \psi\right)$,

where $A, \beta$, and $s$ are phenomenological parameters of the spacer.

From Eq. (3) in a trivial manner we obtain

$$
i \hbar \frac{\partial \psi}{\partial t}=A \Delta \psi-\beta \psi-s\left(\psi^{+} \psi\right) \psi+\mu_{0} H \hat{\sigma}_{z} \psi
$$

for the order-parameter function, which depends on time and coordinates inside the spacer. The boundary conditions have the form

$$
\left.\psi\right|_{z=0}=\text { const, }\left.\psi\right|_{z=L}=\text { const, }
$$

where the points $z=0$ and $z=L$ are the coordinates of the interfaces (see Fig. 1). The solution of Eq. (5) is sought in the form

$$
\psi=\left(\begin{array}{l}
\Psi_{1}(\mathbf{r}) \exp \left(i \omega_{1} t\right) \\
\Psi_{2}(\mathbf{r}) \exp \left(i \omega_{2} t\right)
\end{array}\right) .
$$

By inserting Eq. (7) into Eq. (3) one obtains the system of nonlinear equations which defines the dependence of $\psi_{1}$ and $\psi_{2}$ on the space coordinates $\mathbf{r}$ :

$$
\left\{\begin{array}{l}
\Delta \psi_{1}+a_{1} \psi_{1}-b\left(\left|\psi_{1}\right|^{2}+\left|\psi_{2}\right|^{2}\right) \psi_{1}+h \psi_{1}=0 \\
\Delta \psi_{2}+a_{2} \psi_{2}-b\left(\left|\psi_{1}\right|^{2}+\left|\psi_{2}\right|^{2}\right) \psi_{2}-h \psi_{2}=0
\end{array},\right.
$$

where $a_{i}=\left(\hbar \omega_{i}-\beta\right) / \mathrm{A} ; b=s / A ; h=\mu_{0} H / A$.

For simplicity of consideration we suppose that the external magnetic field $H$ changes the directions of the magnetizations of the MOLs in the same way, i.e., the polar angle $\theta_{0}=\theta_{0}(H)$ for the MOL magnetizations is the same, and the difference in their orientation is described only by change of azimuth angle $\varphi$. Let's choose the constants in the boundary conditions (6) taking into account that the magnetic moment density of the spacer at the interfaces must be parallel or antiparallel, respectively, to the direction of magnetization of the MOLs on variation of the sign of the exchange coupling. Also we suppose that exchange coupling between the magnetization in the spacer and the magnetizations of the MOLs is appreciable only within the interfacial regions $(a<L L)$, i.e., that MOLs influence the spacer only through the boundary conditions (6). Let's consider that the magnetization in the spacer at the boundaries with the MOLs is parallel to the magnetization of the MOLs. The case when the orientation is antiparallel can be considered analogously. In accordance with the above-said, one can write the boundary condition using a spherical co- 


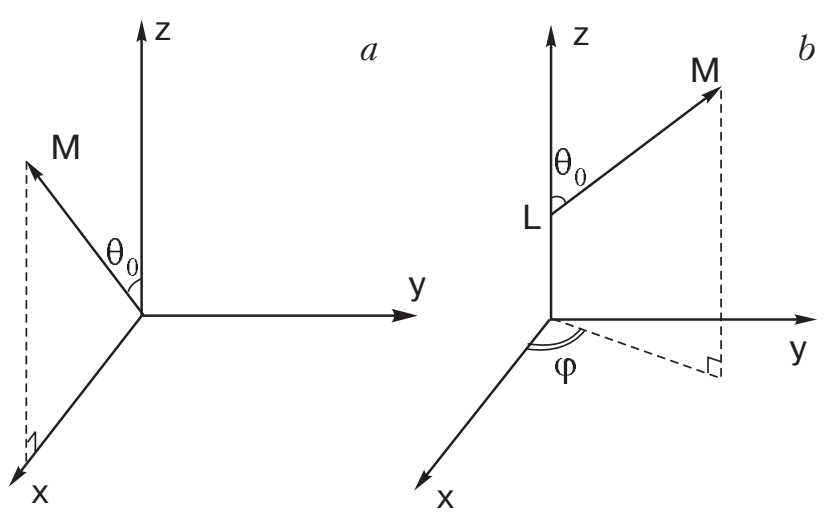

Fig. 1. Direction of magnetization of the spacer at the boundary with the first MOL, $z=0(a)$ and with the boundary with the second MOL, $z=L(b)$.

ordinate system (see Fig. 1) and Pauli matrices, in the form

$$
\begin{gathered}
\left\{\begin{array}{l}
M_{0} \sin \theta_{0}=\left.\mu_{0}\left(\Phi_{1}^{*} \Phi_{2}+\Phi_{2}^{*} \Phi_{1}\right)\right|_{z=0} \\
0=-\left.i \mu_{0}\left(\Phi_{1}^{*} \Phi_{2}-\Phi_{2}^{*} \Phi_{1}\right)\right|_{z=0} \\
M_{0} \cos \theta_{0}=\left.\mu_{0}\left(\Phi_{1}^{*} \Phi_{1}-\Phi_{2}^{*} \Phi_{2}\right)\right|_{z=0}
\end{array}\right. \\
\left\{\begin{array}{l}
M_{0} \sin \theta_{0} \cos \varphi=\left.\mu_{0}\left(\Phi_{1}^{*} \Phi_{2}+\Phi_{2}^{*} \Phi_{1}\right)\right|_{z=L} \\
M_{0} \sin \theta_{0} \sin \varphi=-\left.i \mu_{0}\left(\Phi_{1}^{*} \Phi_{2}-\Phi_{2}^{*} \Phi_{1}\right)\right|_{z=L} \\
M_{0} \cos \theta_{0}=\left.\mu_{0}\left(\Phi_{1}^{*} \Phi_{1}-\Phi_{2}^{*} \Phi_{2}\right)\right|_{z=L}
\end{array}\right.
\end{gathered}
$$

where $M_{0}$ is the magnitude of the magnetization at the boundaries, which plays the role of a phenomenological parameter of our problem. The magnitude $|\mathbf{M}|$ is chosen the same on both interfaces, equal to $M_{0} \approx \mu_{0} / V_{0}$ (where $V_{0}$ is the unit cell vol- ume of near the interface of a spacer layer of thickness $\left.a, V_{0}=L_{x} L_{y} a\right)$, taking into account the assumption of the strong polarizing effect of MOLs on the magnetization of the spacer in the immediate neighborhood of the interface.

It's easy to see using direct substitution, that

$$
\psi=\left(\begin{array}{l}
B_{1} \mathrm{e}^{i \delta_{1}} \mathrm{e}^{i \kappa_{1} \rho} \mathrm{e}^{-i k_{1} z} \mathrm{e}^{i \omega_{1} t} \\
B_{2} \mathrm{e}^{i \delta_{2}} \mathrm{e}^{i \kappa_{2} \rho} \mathrm{e}^{i k_{2} z} \mathrm{e}^{i \omega_{2} t}
\end{array}\right),
$$

where $\kappa_{i}, \delta_{i}$, and $\omega_{i}$ are real magnitudes, and $\rho=(x, y)$ satisfies the system of nonlinear equations (8) if the following correlations between parameters are fulfilled:

$$
\left\{\begin{array}{l}
-\left(\kappa_{1}^{2}+k_{1}^{2}\right)+a_{1}-b\left(B_{1}^{2}+B_{2}^{2}\right)+h=0 \\
-\left(\kappa_{2}^{2}+k_{2}^{2}\right)+a_{2}-b\left(B_{1}^{2}+B_{2}^{2}\right)-h=0
\end{array}\right. \text {. }
$$

To make the solution (11) satisfy the boundary conditions, it is necessary that

$$
\begin{gathered}
\left\{\begin{array} { l } 
{ \omega _ { 1 } = \omega _ { 2 } = \omega } \\
{ \kappa _ { 2 } = \kappa _ { 1 } = \kappa , } \\
{ \delta _ { 2 } - \delta _ { 1 } = \varphi _ { 0 } }
\end{array} \quad \left\{\begin{array}{l}
B_{1}=\sqrt{m_{0}} \cos \frac{\theta_{0}}{2} \\
B_{2}=\sqrt{m_{0}} \sin \frac{\theta_{0}}{2}
\end{array},\right.\right. \\
k_{1}+k_{2}=\frac{\varphi+2 N \pi}{L},
\end{gathered}
$$

where the conditions

$$
N=0, \pm 1, \ldots, \quad m_{0}=\frac{M_{0}}{\mu_{0}}
$$

are fulfilled, too. Then the solution satisfying the system of nonlinear equations (8) and the boundary conditions (9), (10) is transformed to the form

$$
\begin{gathered}
\psi=\sqrt{m_{0}} \mathrm{e}^{i \omega t} \mathrm{e}^{i(\kappa \rho+\delta)}\left(\begin{array}{l}
\cos \frac{\theta_{0}}{2} \exp \left(-i\left\{\frac{\varphi+2 N \pi}{2 L}+\frac{\mu_{0} H L}{A(\varphi+2 N \pi)}\right\} z\right) \\
\sin \frac{\theta_{0}}{2} \exp \left(i\left\{\frac{\varphi+2 N \pi}{2 L}-\frac{\mu_{0} H L}{A(\varphi+2 N \pi)}\right\} z\right)
\end{array}\right), \text { if } \varphi \neq 0, H>0, N=0, \pm 1, \ldots \\
\psi=\sqrt{m_{0}} \mathrm{e}^{i \omega t} \mathrm{e}^{i(\kappa \rho+\delta)}\left(\begin{array}{l}
\left.\cos \frac{\theta_{0}}{2} \exp \left(-i\left\{\frac{N \pi}{L}+\frac{\mu_{0} H L}{2 A N \pi}\right\} z\right)\right) \\
\sin \frac{\theta_{0}}{2} \exp \left(i\left\{\frac{N \pi}{L}-\frac{\mu_{0} H L}{2 A N \pi}\right\} z\right)
\end{array}\right), \text { if } \varphi=0, H>0, N= \pm 1, \pm 2, \ldots
\end{gathered}
$$

By inserting the solution (15) into the correlation (12) one obtains

$$
\varepsilon_{N}=\beta+m_{0} s+A \kappa^{2}+A\left(\frac{\varphi+2 N \pi}{2 L}\right)^{2}+A^{-1}\left(\frac{\mu_{0} H L}{\varphi+2 N \pi}\right)^{2}
$$


where $\varepsilon_{N}=\hbar \oplus$. Let us assume, that Eq. (15) describes the states of quasiparticles (which we will call magnetized electrons), which behave according to Fermi-Dirac statistics. According to Ref. 22 let us define the total number of magnetized electrons in the spacer magnetopolarized by the MOLs, $N_{0}$ as a sum over all possible states of the distribution function. Let's consider, for simplicity, the case when $T=0$, because the most interesting magnetoresistive properties of multilayered nanostructured systems appear at low temperatures. Then the Fermi-Dirac distribution function has the form

$$
f_{0}\left(\varepsilon_{N}\right)=\left\{\begin{array}{ll}
1, & \varepsilon_{N} \leq \varepsilon_{F} \\
0, & \varepsilon_{N}>\varepsilon_{F}
\end{array},\right.
$$

where $\varepsilon_{F}$ is the Fermi energy of the magnetized electrons. According to Eq. (17), the total number of magnetized electrons in the spacer is written in the form

$$
N_{0}=2 \frac{L_{x} L_{y}}{(2 \pi)^{2}} \sum_{N} \int_{0}^{\kappa_{N}} 2 \pi \kappa d \kappa
$$

where $L_{x} \approx L_{y} \gg L$ are the dimensions of the spacer in the $x y$ plane, and $\kappa_{N}$ is the largest possible value of the wave vector $\kappa$ when $N$ is fixed. $\kappa$ is calculated as

$\kappa_{N}=\sqrt{\frac{\varepsilon_{F}-\beta-m_{0} s}{A}-\left(\frac{\varphi+2 N \pi}{2 L}\right)^{2}-\left(\frac{\mu_{0} H L}{A(\varphi+2 N \pi)}\right)^{2}}$

It is easy to obtain from Eq. (13) that

$$
n L=\frac{1}{2 \pi} \sum_{N} \kappa_{N}^{2},
$$

where $n=N_{0} /\left(L_{x} L_{y} L\right)$ is the density of magnetized electrons in the spacer. The components of the specific magnetization of magnetized electrons in a plane with an arbitrary coordinate $z$ inside the spacer, taking into account Eq. (2), have the form:

$$
\left\{\begin{array}{l}
M_{x N}=M_{0} \sin \theta_{0} \cos \left(\frac{\varphi+2 N \pi}{L} z\right) \\
M_{y N}=M_{0} \sin \theta_{0} \sin \left(\frac{\varphi+2 N \pi}{L} z\right) . \\
M_{z N}=M_{0} \cos \theta_{0}
\end{array}\right.
$$

The average $x$ component of the specific magnetization of the near-interface spacer layer of thickness $a$ equals

$$
\left\langle M_{x N}\right\rangle=L_{x} L_{y} \int_{0}^{a} M_{0} \sin \theta_{0} \cos \left(\frac{\varphi+2 N \pi}{L} z\right) d z=
$$

$$
=\frac{L L_{x} L_{y} M_{0} \sin \theta_{0} \sin \left(\frac{\varphi+2 N \pi}{L} a\right)}{\varphi+2 N \pi} .
$$

The average $y$ component of the specific magnetization of the near-interface spacer layer of thickness $a$ equals

$$
\begin{gathered}
\left\langle M_{y N}\right\rangle=L_{x} L_{y} \int_{0}^{a} M_{0} \sin \theta_{0} \sin \left(\frac{\varphi+2 N \pi}{L} z\right) d z= \\
=-\frac{L L_{x} L_{y} M_{0} \sin \theta_{0} \cos \left(\frac{\varphi+2 N \pi}{L} a\right)}{\varphi+2 N \pi} .
\end{gathered}
$$

The average $z$ component of the specific magnetization of the near-interface spacer layer of thickness $a$ accordingly equals

$$
\left\langle M_{z N}\right\rangle=L_{x} L_{y} M_{0} a \cos \theta_{0} .
$$

In the limiting case when $a<<L$, the average absolute value of the magnetization of near-interface spacer layer, $\langle M\rangle=\sqrt{\left\langle M_{x N}\right\rangle^{2}+\left\langle M_{y N}\right\rangle^{2}+\left\langle M_{z N}\right\rangle^{2}}$, equals $L_{x} L_{y} M_{0} a$. With the help of Eq. (17) one can find the magnetization of the near-interface spacer layer of thickness $a$ :

$$
M=2 \frac{L_{x} L_{y} M_{0}}{(2 \pi)^{2}} \sum_{N} \int_{0}^{\kappa_{N}} 2 \pi \kappa d \kappa
$$

Taking into account Eq. (20), one can find that $M=M_{0} n L_{x} L_{y} L$. Using the ratio $M_{0}=\mu_{0} /\left(L_{x} L_{y} a\right)$, one can write

$$
M=\frac{\mu_{0} n L}{a},
$$

hence:

$$
n=\frac{M a}{\mu_{0} L} .
$$

We denote $n_{0}=M / \mu_{0}, n=a_{0} / L$, and $a_{0}=M a / \mu_{0}$ is a phenomenological constant.

Taking into account Eqs. (19) and (20), one obtains a transcendental algebraic equation for finding the Fermi energy $\varepsilon_{F}$ :

$$
\begin{gathered}
n L=\frac{1}{2 \pi} \sum_{N}\left\{\frac{\varepsilon_{F}-\beta-m_{0} s}{A}-\left(\frac{\varphi+2 N \pi}{2 L}\right)^{2}-\right. \\
\left.-\left(\frac{\mu_{0} H L}{A(\varphi+2 N \pi)}\right)^{2}\right\},
\end{gathered}
$$

where the allowed values of $N$ are found from the inequality $\kappa_{N}^{2} \geq 0 . N$ belongs to 


$$
N \in\left[- \text { floor }\left\{\frac{\varphi}{2 \pi}+\frac{\mu_{2}}{2 \pi}\right\} ;-\operatorname{ceil}\left\{\frac{\varphi}{2 \pi}+\frac{\mu_{1}}{2 \pi}\right\}\right] \cup\left[\operatorname{ceil}\left\{-\frac{\varphi}{2 \pi}+\frac{\mu_{1}}{2 \pi}\right\} ; \text { floor }\left\{-\frac{\varphi}{2 \pi}+\frac{\mu_{2}}{2 \pi}\right\}\right] \text {, }
$$

where

$$
\begin{aligned}
& \mu_{1}=\sqrt{2} L \sqrt{\frac{\varepsilon_{F}-\beta-m_{0} s}{A}-\sqrt{\left(\frac{\varepsilon_{F}-\beta-m_{0} s}{A}\right)^{2}-\left(\frac{\mu_{0} H}{A}\right)^{2}}}, \\
& \mu_{2}=\sqrt{2} L \sqrt{\frac{\varepsilon_{F}-\beta-m_{0} s}{A}+\sqrt{\left(\frac{\varepsilon_{F}-\beta-m_{0} s}{A}\right)^{2}-\left(\frac{\mu_{0} H}{A}\right)^{2}}},
\end{aligned}
$$

ceil is the function that gives the maximal integer number closest to a given real number, and floor is the function that gives the minimal integer number closest to a given real number. The average energy of the spacer is defined by the standard formula

$$
\langle\varepsilon\rangle=2 \frac{L_{x} L_{y}}{(2 \pi)^{2}} \sum_{N} \int_{0}^{\kappa_{N}} 2 \pi \kappa \varepsilon_{N}(\kappa) d \kappa
$$

After simple transformations, Eq. (32) transforms to:

$$
\begin{gathered}
\frac{\langle\varepsilon\rangle}{L_{x} L_{y}}=\left(\beta+m_{0} s\right) n L+ \\
+\frac{A}{4 \pi} \sum_{N}\left\{\left(\frac{\varepsilon_{F}-\beta-m_{0} s}{A}\right)^{2}-\left(\frac{\varphi+2 N \pi}{2 L}\right)^{4}-\right. \\
\left.-\left(\frac{\mu_{0} H L}{A(\varphi+2 N \pi)}\right)^{4}-\frac{1}{2}\left(\frac{\mu_{0} H}{A}\right)^{2}\right\}
\end{gathered}
$$

where $\langle\varepsilon\rangle /\left(L_{x} L_{y}\right)$ is the spacer's energy per a unit area.

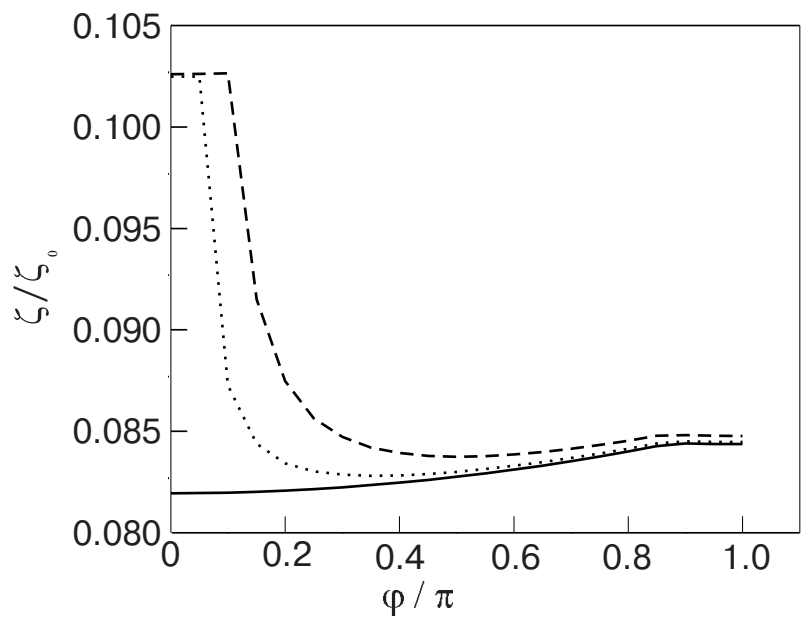

Fig. 2. Dependence of Fermi energy on the angle $\varphi$. Dashed line $H=800 \mathrm{Oe}$, dotted line $H=400 \mathrm{Oe}$, solid line $H=0$ Oe. $\zeta_{0}=A\left(3 \pi^{2} N\right)^{2 / 3}, \zeta=\varepsilon_{F}-\beta-m_{0} s, L=23 \AA$.
By comparing the experimental data for the exchange coupling energy between MOLs and the maximal exchange coupling energy between the layers [23-29] for different types of trilayer systems with the results of numerical calculations using the given model, it was found that if the density of magnetized electrons $n$ equals the tabulated point for the corresponding metal spacer, then the parameter $a \approx 1 \AA$ and the parameter $A$ may occupy the region $10^{-30} \mathrm{erg} \cdot \mathrm{cm}^{2}<A<10^{-27} \mathrm{erg} \cdot \mathrm{cm}^{2}$. For example, Eq. (28) was solved numerically for the following values of the spacer's parameters: $A=0.15 \cdot 10^{-30} \mathrm{erg} \cdot \mathrm{cm}^{2}$, $n=5,9 \cdot 10^{22} \mathrm{~cm}^{-3}, \theta_{0}=\pi / 2, a=1 \AA$. The curves of the Fermi energy as a function of the spacer's thickness, on magnetic field, and on the angle $\varphi$, while the remaining parameters are fixed, are shown, are performed in Fig. 2, 3, and 4, respectively. It is obvious from the plots that the Fermi energy has oscillating dependence on the spacer's thickness and on magnetic field. These oscillations are a purely dimensional effect analogous to that described Ref. 22.

The results of numerical calculation of $\langle\varepsilon\rangle /\left(L_{x} L_{y}\right)$ for the spacer's parameters, taking into account calculated numerically Fermi energy, are shown in Figs. 5

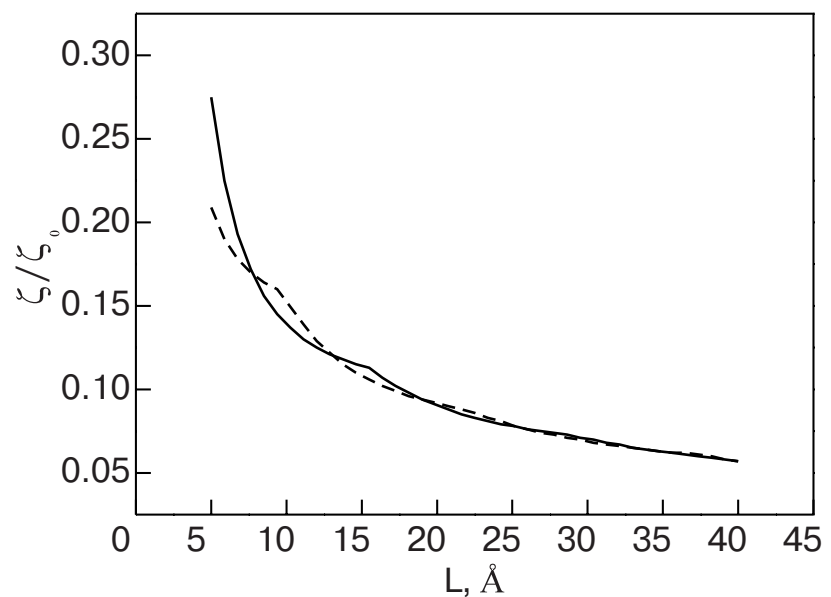

Fig. 3. Dependence of Fermi energy on the spacer's thickness. Solid line $\varphi=0$, dashed line $\varphi=\pi, H=0$. 


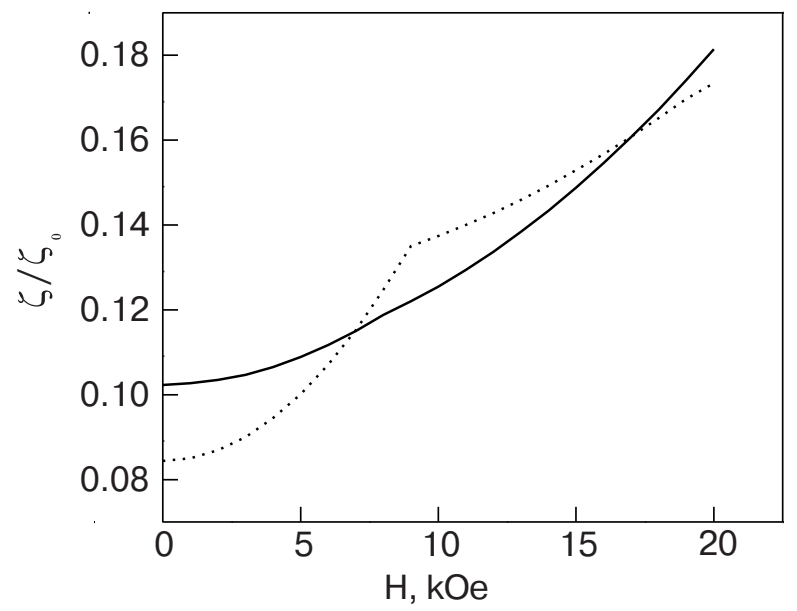

Fig. 4. Dependence of the Fermi energy on magnetic field. Solid line $\varphi=0$, dotted line $\varphi=\pi, L=23 \AA$.

and 6. It is obvious from the Figs. 5 and 6 that both $\langle\varepsilon\rangle /\left(L_{x} L_{y}\right)$ and $\varepsilon_{F}$ also have oscillating behavior depending on the spacer's thickness and magnetic field. $\langle\varepsilon\rangle /\left(L_{x} L_{y}\right)$ and $\varepsilon_{F}$ also have minima when $\varphi=0$ or $\varphi=\pi$, depending on spacer thickness, magnetic field, and other parameters. That is, the energy-optimal configuration is the parallel or antiparallel mutual orientation of the projections of the magnetizations of the MOLs on the $x y$ plane.

As is seen from Fig. 7, the exchange coupling of the MOLs through the metal spacer has a long-periodic oscillatory dependence on the spacer's thickness $L$, i.e., the exchange coupling changes from antiferromagnetic to ferromagnetic depending on the spacer's thickness. As is seen from Fig. 5, the given theore-

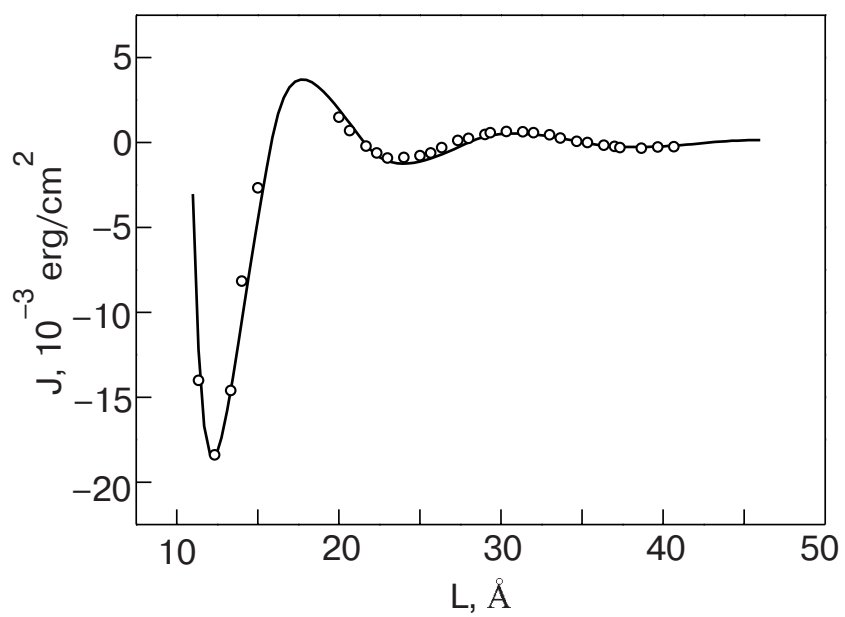

Fig. 5. Dependence of exchange coupling energy $J=\langle\varepsilon\rangle /\left.\left(L_{x} L_{y}\right)\right|_{\varphi=\pi}-\langle\varepsilon\rangle /\left.\left(L_{x} L_{y}\right)\right|_{\varphi=0}$ on the spacer's thickness. Solid line is theoretical calculation, «o» are the results of the experimental work [29]. It was found by comparing these theoretical and experimental results that they are shifted by $6 \AA$.

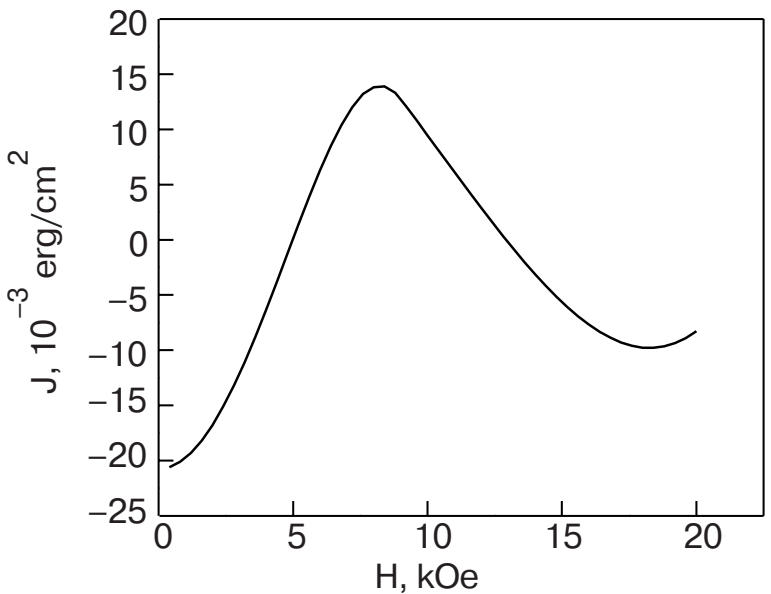

Fig. 6. Dependence of the exchange coupling energy $J$ on magnetic field. $L=23 \AA$.

tical calculation shows that $J=\langle\varepsilon\rangle /\left.\left(L_{x} L_{y}\right)\right|_{\varphi=\pi}-$ - $\langle\varepsilon\rangle /\left.\left(L_{x} L_{y}\right)\right|_{\varphi=0}$ descends faster than $L^{-2}$ with increase of $L$. Moreover, the period of the oscillations of $J$ is not constant but increases with increase of $L$. Both of these theoretical results agree quantitatively with results of Ref. 29 (see Fig. 5). As is seen from Figs. 8 and 9 , the theoretically obtained values of the first three periods of oscillation of the exchange coupling equal 10,12 , and $15 \AA$, respectively. The experimentally obtained periods of oscillation of the exchange coupling (according to Ref. 29) equal to 11.0, 13.6 and $15.2 \AA$ with accuracy $\pm 1 \AA$.

The components of the magnetization at a point with an arbitrary coordinate $z$ inside the spacer have the form (21). Then, taking into account the Fermi-Dirac statistics and the distribution functions (17), the mean value of the magnetization components

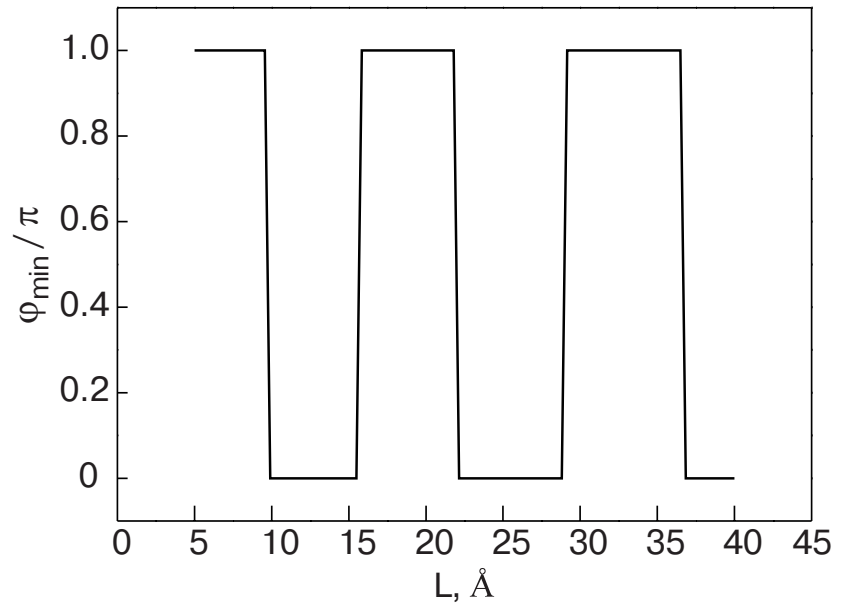

Fig. 7. Dependence of the angle when the energy of coupling of the MOLs is minimal as a function of the spacer's thickness. $H=0$. 


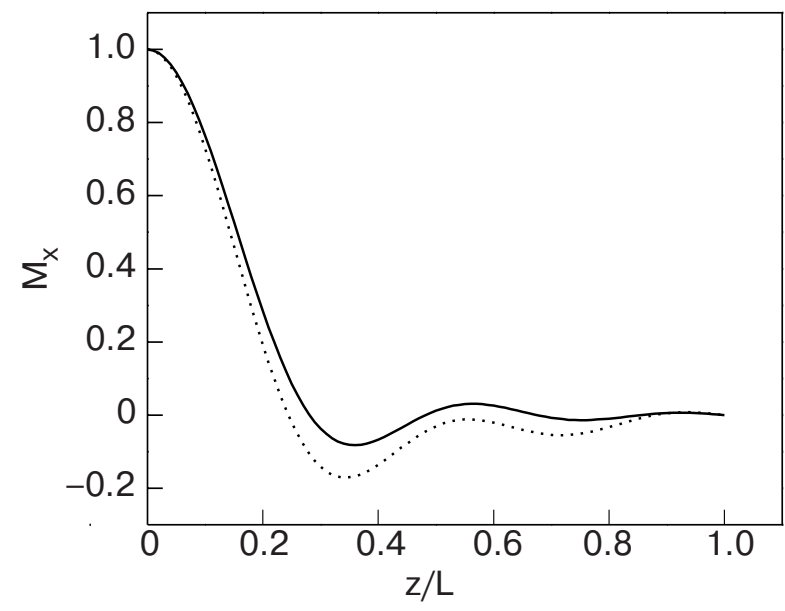

Fig. 8. Magnetization component of $M_{x}$ (divided by the magnetization at the $z=0$ boundary) as a function of $z$. Solid line $H=0$ Oe, dotted line $H=2000$ Oe. The spacer's thickness is $23 \AA$.

at an arbitrary point with coordinate $z$ inside the spacer has the form:

$$
\left\{\begin{array}{l}
\left\langle M_{x}\right\rangle=2 \frac{L_{x} L_{y}}{(2 \pi)^{2}} \sum_{N} \int_{0}^{\kappa_{N}} 2 \pi \kappa d \kappa M_{x N} \\
\left\langle M_{y}\right\rangle=2 \frac{L_{x} L_{y}}{(2 \pi)^{2}} \sum_{N} \int_{0}^{\kappa_{N}} 2 \pi \kappa d \kappa M_{y N} \\
\left\langle M_{z}\right\rangle=2 \frac{L_{x} L_{y}}{(2 \pi)^{2}} \sum_{N} \int_{0}^{\kappa_{N}} 2 \pi \kappa d \kappa M_{z N}
\end{array}\right.
$$

It is simple to transform Eq. (34) to the form:

$$
\begin{gathered}
\frac{\left\langle M_{x}\right\rangle}{L_{x} L_{y}}=\frac{1}{2 \pi} \sum_{N} M_{0} \sin \theta_{0} \cos \left(\frac{\varphi+2 N \pi}{L} z\right) \times \\
\times\left\{\frac{\varepsilon_{F}-\beta-m_{0} s}{A}-\left(\frac{\varphi+2 N \pi}{2 L}\right)^{2}-\left(\frac{\mu_{0} H L}{A(\varphi+2 N \pi)}\right)^{2}\right\}, \\
\times\left\{\frac{\left\langle M_{y}\right\rangle}{L_{x} L_{y}}=\frac{1}{2 \pi} \sum_{N} M_{0} \sin \theta_{0} \sin \left(\frac{\varphi+2 N \pi}{L} z\right) \times\right. \\
A \quad-m_{0} s \\
\left.\frac{\varepsilon_{F}}{A}\left(\frac{\varphi+2 N \pi}{2 L}\right)^{2}-\left(\frac{\mu_{0} H L}{A(\varphi+2 N \pi)}\right)^{2}\right\}, \\
\frac{\left\langle M_{z}\right\rangle}{L_{x} L_{y}}=M_{0} \cos \theta_{0} n L .
\end{gathered}
$$

The absolute value of the magnetization at a point with coordinate $z$ inside the spacer is calculated as

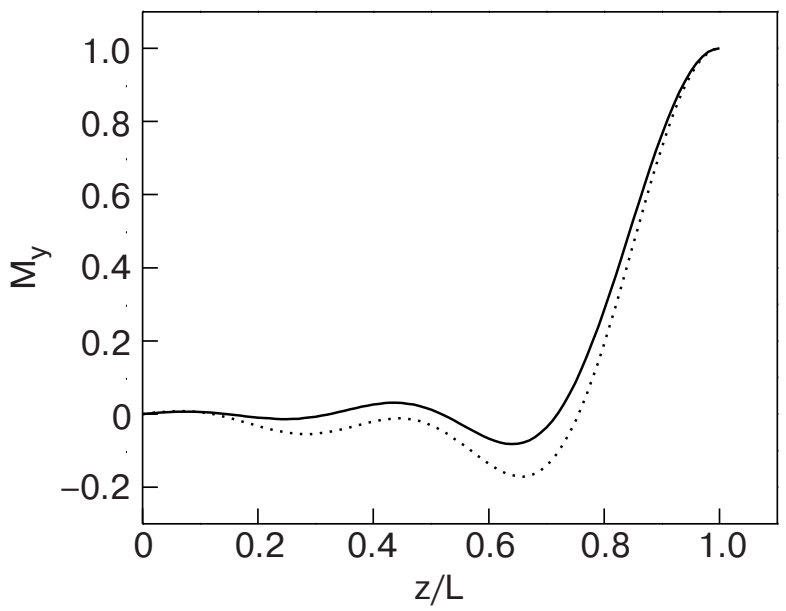

Fig. 9. Magnetization component of $M_{y}$ (divided by magnetization at the $z=L$ boundary) as a function of $z$. Solid line $H=0$ Oe, dotted line $H=2000$ Oe. The $H=2000$ Oe spacer's thickness is $23 \AA$.

$$
\langle|M|\rangle=\sqrt{\left\langle M_{x}\right\rangle^{2}+\left\langle M_{y}\right\rangle^{2}+\left\langle M_{z}\right\rangle^{2}} .
$$

The plots of the dependences of $\left\langle M_{x}\right\rangle$ and $\left\langle M_{y}\right\rangle$ on coordinate $z$ inside the spacer are performed in Figs. 8 and 9. Here the boundary conditions are chosen so that when $z=0$, the magnetization of the spacer is directed along the $x$ axis, and when $z=L$ the magnetization of the spacer is directed along the $y$ axis. The magnitude $\left\langle M_{z}\right\rangle$, as is seen from Eq. (37), is constant at all spacer thicknesses, according to our model. As seen from Fig. 10, the average value of $|M|$ is maximal on the spacer's boundaries with the MOLs and decays from the spacer's boundaries inside the spacer symmetrically about of the spacer middl.

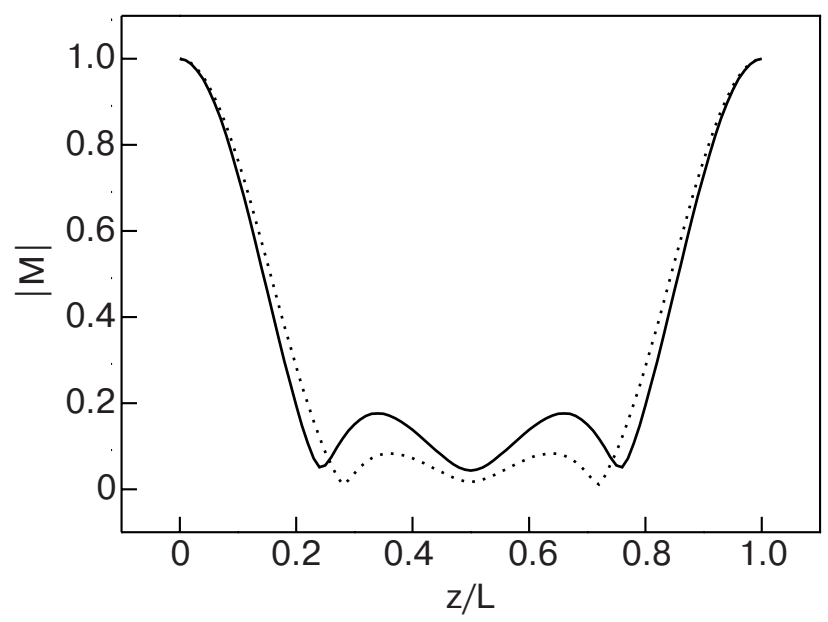

Fig. 10. Absolute value of $M$ (divided by the magnetization at the $z=0$ boundary) as a function of $z$. Solid line $H=0$ Oe, dotted line $H=2000$ Oe. The spacer's thickness is $23 \AA$. 


\section{Discussion of results}

In this paper the exchange coupling of magnetically ordered layers through a nonmagnetic metallic spacer was calculated using a spin-density model similar to the Ginzburg-Landau model. The induced magnetization in the spacer, taking into account the influence of an external magnetic field, was calculated, too. This calculation shows that the energy of coupling of the MOLs through the nonmagnetic metallic spacer is an oscillating function of the spacer's thickness and magnetic field, i.e., the exchange coupling between the layers varies from ferromagnetic to antiferromagnetic and vice versa in variation of the spacer's thickness and magnetic field. Here the magnitude of the exchange coupling decreases with spacer thickness faster than $L^{-2}$ and the period of oscillation of the exchange coupling is not constant but increaeses with increase of spacer thickness. These results match with the works [29-31], which were carried out for $\mathrm{Cu}$ and $\mathrm{Au}$ spacers and for Co and Fe MOLs. Also, this calculation shows that in a nonferromagnetic spacer the induced magnetization can undergo many complete rotations with variation of distance to the boundaries with the MOLs. Moreover, the absolute value of the induced magnetization decays nonmonotonically with distance from the interfaces inside the spacer. It is shown that the character of the decay of the absolute value of the magnetization from the interfaces into the interior of the spacer is influenced by magnetic field.

The author is grateful to Prof. V.G. Baryakhtar for fruitful discussion of the results and to Prof. A.V. Svidzinsky for discussion of basic aspects of the model used in this work for calculation of the induced magnetization of the spacer.

1. S.S.P. Parkin, Phys. Rev. Lett. 71, 1641 (1993).

2. P. Bruno, Phys. Rev. B52, 411 (1995).

3. S.S.P. Parkin, N. More, and K.P. Roche, Phys. Rev. Lett. 64, 2304 (1990).

4. W.R. Bennett, W. Schwarzacher, and W.F. Egelhoff, Jr., Phys. Rev. Lett. 65, 3169 (1990).

5. D.H. Mosca, F. Petroff, A. Fert, P.A. Schroeder, W.P. Pratt Jr., and R. Laloee, J. Magn. Magn. Mater. 94, L1 (1991).

6. S.S.P. Parkin, R. Bhadra, and K.P. Roche, Phys. Rev. Lett. 66, 2152 (1991).

7. S.S.P. Parkin, Phys. Rev. Lett. 67, 3598 (1991).

8. L.M. Falicov, Daniel T. Pierce, S.D. Bader, Kristl B. Hathaway, Herbert J. Hopster, David N. Lambeth, S.S.P. Parkin, Gary Prinz, Myron Salamon, Ivan
K. Schuller, and R.H. Victora, J. Mater. Res. 5, 1299 (1990).

9. P.W. Rooney, A.L. Shapiro, M.Q. Tran, and F. Hellman, Phys. Rev. Lett. 75, 1843 (1995).

10. G.P. Felcher, R.O. Hilleke, R.K. Crawford, J. Haumann, R. Kleb, and G. Ostrowsky, Rev. Sci. Instrum. 58, 609 (1987).

11. S. Hamada, N. Hosoito, and T. Shinjo, J. Phys. Soc. Jpn. 66, 30 (1997).

12. N. Hosoito, T. Emoto, S. Hamada, and T. Shinjo, ICR Annual Report 3, 167 (1996).

13. M.G. Samant, J. Stoehr, S.S.P. Parkin, G.A. Held, B.D. Hermsmeier, F. Herman, M. van Schilfgaarde, L.C. Duda, D.C. Mancini, N. Wassdahl, and R. Nakajima, Phys. Rev. Lett. 72, 1112 (1994).

14. S. Pizzini, A. Fontaine, Ch. Giorgetti, E. Dartige, J.F. Bobo, M. Piecuch, and F. Baudelet, Phys. Rev. Lett. 74, 1470 (1995).

15. Q.Y. Jin, Y.B. Xu, H.R. Zhai, C. Hu, M. Lu, Q.S. Bie, Y. Zhai, G.L. Dunifer, R. Naik, and M. Ahmad, Phys. Rev. Lett. 72, 768 (1994).

16. W. Harrison, Solid State Theory, Mir, Moscow (1972).

17. J. Mathon, A. Umerski, M. Milleret, R.B. Muniz, and D.M. Edwards, J. Magn. Magn. Mater. 217, 188 (2000).

18. L. Nordstroem and D.J. Singh, J. Appl. Phys. 79, 4515 (1996).

19. J. Mathon, A. Umerski, M. Milleret, and R.B. Muniz, Phys. Rev. B59, 6344 (1999).

20. V.G. Baryakhtar and Yu.I. Gorobets, Bubble Magnetic Domains and their Lattices, Naukova Dumka, Kiev (1988).

21. E.M. Lifshitz and L.P. Pitayevsky, Statistical Physics, Nauka, Moscow (1978).

22. V. Bezak, J. Phys. Chem. Solids 27, 815 (1966).

23. P. Bruno, Phys. Rev. B52, 411 (1995).

24. S.O. Demokritov, J. Phys. D31, 925 (1998).

25. P. Grünberg, R. Schreiber, Y. Pang, M.B. Brodsky, and H. Sowers, Phys. Rev. Lett. 57, 2442 (1986).

26. C.F. Majkrzak, J.W. Cable, J. Kwo, M. Hong, D.B. Mc Whan, Y. Yafet, J.V. Waszczak, and C. Vettier, Phys. Rev. Lett. 56, 2700 (1986).

27. C.F. Majkrzak, J. Kwo, M. Hong, Y. Yafet, Doon Gibbs, C.L. Chien, and J. Bohr, Adv. Phys. 40, 99 (1991).

28. S.S.P. Parkin, N. More, and K.P. Roche, Phys. Rev. Lett. 64, 2304 (1990).

29. J.J. de Vries, P.J.H. Bloemen, M.T. Johnson, J. van de Stegge, A. Reinders, and W.J.M. de Jonge, J. Magn. Magn. Mater. 129, L129 (1994).

30. K. Shintaku, Y. Daitoh, and T. Shinjo, Phys. Rev. B47, 14584 (1993).

31. M.T. Johnson, S.T. Purcell, N.W.E. McGee, R. Coehoorn, J aan de Stegge, and W. Hoving, Phys. Rev. Lett. 68, 2688 (1992). 